\title{
SYMMETRY ANALYSIS OF ROTATING FLUID
}

\author{
K. FAKHAR ${ }^{\varpi 1}$, ZU-CHI CHEN ${ }^{1}$ and XIAODA JI ${ }^{1}$
}

(Received 11 June, 2004)

\begin{abstract}
The machinery of Lie theory (groups and algebras) is applied to the unsteady equations of motion of rotating fluid. A special-function type solution for the steady state is derived. It is then shown how the solution generates an infinite number of time-dependent solutions via three arbitrary functions of time. This algebraic structure also provides the mechanism to search for other solutions since its character is inferred from the basic equations.
\end{abstract}

\section{Introduction}

The unsteady flow created by a rotating fluid has attracted the attention of many researchers. Much of this interest is because of the possible implications of this type of flow for flows in general. However, the solutions of many practical rotating flow problems hinge on an understanding of the behaviour of the unsteady boundary layers. Mention should be made of the interesting work of Greenspan and Howard [8], who initiated the study of the dynamics of spin-up of an incompressible homogeneous viscous rotating fluid. Following in the footsteps of Greenspan and Howard's groundbreaking work [8], several researchers such as Holton [10], Walin [16] and Siegmann [15] have shown that the spin-up mechanism for a stratified fluid is different to and much more complicated than the corresponding process for a homogeneous fluid. In order to study the transient motion in the earth's liquid core, Debnath [2-4] examined a hydromagnetic spin-up mechanism in an electrically conducting rotating viscous fluid in the presence of an external magnetic field. In another paper, Debnath and Mukherjee [5] discussed the unsteady multiple boundary layers on a porous plate in a rotating system.

The basic governing equations describing the rotating fluid phenomenon are nonlinear in nature. The perturbation technique is that which is widely used by physicists

\footnotetext{
${ }^{1}$ Department of Mathematics, University of Science and Technology of China, Hefei, Anhui 230026, P. R. China; e-mail: kamranfakhar@yahoo.com.

(C) Australian Mathematical Society 2005, Serial-fee code 1446-1811/05
} 
and engineers to handle these types of nonlinear physical phenomena. We have obtained many interesting and important results by utilising this technique. However, the perturbation methods have their own limitations such as the fact that all perturbation techniques are based on small or large parameters so that at least one unknown must be expressed in a series of small parameters. Unfortunately, not every nonlinear differential equation has this kind of structure. Even if such a small parameter exists, the result given by perturbation methods is valid, in most cases, only for small values of the parameter. Mostly, the simplified linear equations have different properties from the original nonlinear differential equation, and sometimes some initial or boundary conditions are superfluous for the simplified linear equations. As a result, the corresponding initial approximations may be far from exact. Clearly, these limitations of perturbation techniques arise from the small parameter assumption. So it seems necessary to use another method which does not require these kinds of assumptions.

The Lie symmetry analysis is one such method which does not require small parameter assumptions at all. The primary objective of the Lie symmetry analysis advocated by Sophus Lie is to find one- or several-parameter local continuous transformations leaving the equations invariant and then exploit them to obtain the so-called invariant or similarity solutions, invariants, integrals of motion, etc. [1, 11, 13], and the usefulness of this approach has been widely illustrated by several authors in different contexts $[6,7,9,12,17]$.

In this article, we consider the unsteady motion of the conducting fluid in the rotating Cartesian coordinate system. Lie group theory is applied to the equations of motion in search of similarity solutions. The symmetries of the equations are found. By using these symmetries, a similarity reduction of the equations of motion to an ordinary differential equation is obtained. The resulting differential equation is reduced to a Bessel-type equation. An exact solution containing a special function is constructed. The physical interpretation along with a plot of the solution is presented at the end.

\section{Equations of motion}

Consider a semi-infinite expanse of homogeneous, incompressible, electrically conducting viscous fluid bounded by an infinite non-conducting rigid disk at $z=0$ in the presence of a uniform magnetic field $B_{0}$ normal to the disk. Both the fluid and the disk are in the state of a rigid body rotation with constant angular velocity $\Omega$ about the $z$-axis normal to the disk.

The unsteady motion of the conducting fluid in the rotating Cartesian coordinate system is governed by the laws of conservation of mass and of momentum which are

$$
\frac{\partial V}{\partial t}+(V \cdot \nabla) V+2 \Omega \times V=-\nabla p+\frac{j \times B}{\rho}+\mu \nabla^{2} V
$$




$$
\operatorname{div} V=0 \text {, }
$$

where $V=(u, v, w)$ is the velocity vector, $p$ is the modified pressure including the centrifugal term, $\rho$ is the (constant) density,$j$ is the electric current density, $B$ is the total magnetic field so that $B=B_{0}+b, b$ is the induced magnetic field and $\mu$ is (constant) viscosity. We assume that the velocity field depends on $x, y$ and $t$ with $w=0$ so that $V=[u(x, y, t), v(x, y, t), 0]$. Further, the magnetic field $B$ is perpendicular to the velocity field. In low magnetic field Reynolds-number approximations, the induced magnetic field is negligible compared with the imposed field [14]. Also the electric field is assumed to be zero so only the magnetic field $B_{0}$ contributes to the current $j=\sigma(V \times B)$. Consequently, under these assumptions the electromagnetic body force involved in (2.1) becomes

$$
\frac{j \times B}{\rho}=-\frac{\sigma}{\rho} B_{0}^{2} V=-n^{*} V,
$$

where $\sigma$ is the electrical conductivity of the fluid and $n^{*}=(\sigma / \rho) B_{0}^{2}$ has the same dimension as $\Omega$ and plays an important role in the present hydromagnetics analysis. In view of (2.3), the equations of motion (2.1)-(2.2) in component form become

$$
\begin{aligned}
\frac{\partial u}{\partial t}+u \frac{\partial u}{\partial x}+v \frac{\partial u}{\partial y}-2 \Omega v+n^{*} u & =-\frac{\partial p}{\partial x}+\mu\left(\frac{\partial^{2} u}{\partial x^{2}}+\frac{\partial^{2} u}{\partial y^{2}}\right) \\
\frac{\partial v}{\partial t}+u \frac{\partial v}{\partial x}+v \frac{\partial v}{\partial y}+2 \Omega u+n^{*} v & =-\frac{\partial p}{\partial y}+\mu\left(\frac{\partial^{2} v}{\partial x^{2}}+\frac{\partial^{2} v}{\partial y^{2}}\right) \\
\frac{\partial u}{\partial x}+\frac{\partial v}{\partial y} & =0
\end{aligned}
$$

where $u$ and $v$ are the velocity components.

\section{Symmetry groups and generators of equations}

In order to find invariance transformations, we look for infinitesimal Lie point transformations of the form

$$
\begin{aligned}
t^{*} & =t+\varepsilon \eta_{1}(t, x, y, u, v, p)+O(\varepsilon)^{2}, \\
x^{*} & =x+\varepsilon \eta_{2}(t, x, y, u, v, p)+O(\varepsilon)^{2}, \\
y^{*} & =y+\varepsilon \eta_{3}(t, x, y, u, v, p)+O(\varepsilon)^{2}, \\
u^{*} & =u+\varepsilon \eta_{4}(t, x, y, u, v, p)+O(\varepsilon)^{2}, \\
v^{*} & =v+\varepsilon \eta_{5}(t, x, y, u, v, p)+O(\varepsilon)^{2}, \\
p^{*} & =p+\varepsilon \eta_{6}(t, x, y, u, v, p)+O(\varepsilon)^{2},
\end{aligned}
$$


which leaves (2.4)-(2.6) invariant.

Following widely used classical methods concerning these arguments $[1,11,13]$ we find the infinitesimal symmetries $\eta_{i}, i=1, \ldots, 6$, by solving the over determined linear PDE system, usually called the determining system, obtained by requiring the invariance of (2.4)-(2.6) with respect to (3.1).

There are many software packages may be used to help obtain the determining system. But solving the system with arbitrary functions requires analysis. We omit the determining system from which we are able to obtain the following results for the form of the infinitesimal symmetries:

$$
\begin{aligned}
\eta_{1}= & \alpha, \quad \eta_{2}=\gamma y+f(t), \quad \eta_{3}=-\gamma x+g(t), \\
\eta_{4}= & \gamma v+f^{\prime}(t), \quad \eta_{5}=-\gamma u+g^{\prime}(t), \\
\eta_{6}= & j(t)-x\left[n^{*} f^{\prime}(t)+f^{\prime \prime}(t)-2 \Omega g^{\prime}(t)\right] \\
& -y\left[n^{*} g^{\prime}(t)+g^{\prime \prime}(t)+2 \Omega f^{\prime}(t)\right],
\end{aligned}
$$

where $\alpha$ and $\gamma$ are arbitrary parameters and $j(t), f(t)$ and $g(t)$ are arbitrary sufficiently smooth functions of time. The parameter $\alpha$ corresponds to a translation in $t$ and the parameter $\gamma$ represents rotations of the spatial system. The infinitesimal operator (the generator of Lie algebra) associated with each parameter is obtained from the operator

$$
Q=\eta_{1} \frac{\partial}{\partial t}+\eta_{2} \frac{\partial}{\partial x}+\eta_{3} \frac{\partial}{\partial y}+\eta_{4} \frac{\partial}{\partial u}+\eta_{5} \frac{\partial}{\partial v}+\eta_{6} \frac{\partial}{\partial p},
$$

by setting the studied parameter equal to one while other parameters and arbitrary functions are equated to zero. The operator associated with each of the arbitrary functions in (3.2) is obtained by setting the other arbitrary functions and all parameters identically equal to zero. If $G_{i}, i=1, \ldots, 5$, are the generators associated with the parameters $\alpha$ and $\gamma$ and the arbitrary functions $j(t), f(t)$ and $g(t)$, then we have

$$
\begin{aligned}
G_{1} & =\frac{\partial}{\partial t}, \quad G_{2}=y \frac{\partial}{\partial x}-x \frac{\partial}{\partial y}+v \frac{\partial}{\partial u}-u \frac{\partial}{\partial v}, \\
G_{3}(f(t)) & =f(t) \frac{\partial}{\partial x}+f^{\prime}(t) \frac{\partial}{\partial u}-\left[\left(n^{*} x+2 \Omega y\right) f^{\prime}(t)+x f^{\prime \prime}(t)\right] \frac{\partial}{\partial p}, \\
G_{4}(g(t)) & =g(t) \frac{\partial}{\partial y}+g^{\prime}(t) \frac{\partial}{\partial v}-\left[\left(n^{*} y-2 \Omega x\right) g^{\prime}(t)+y g^{\prime \prime}(t)\right] \frac{\partial}{\partial p}, \\
G_{5} & =j(t) \frac{\partial}{\partial p} .
\end{aligned}
$$

The generators $G_{1}$ and $G_{2}$ generate a finite-dimensional Lie algebra $L_{2}$ which is a two-dimensional subalgebra of the infinite-dimensional algebra $L_{\infty}$ generated by the generators $G_{i}, i=1, \ldots, 5$. 


\section{Solution via subgroup generated by $f(t), g(t)$ and $j(t)$}

In this section, a similarity solution (group invariant solution) corresponding to the above symmetries will be derived. We first consider translation in the $t$ coordinate and hence $\alpha=1$ is taken. This subgroup has the values

$$
\begin{aligned}
& \eta_{1}=1, \quad \eta_{2}=f(t), \quad \eta_{3}=g(t), \quad \eta_{4}=f^{\prime}(t), \quad \eta_{5}=g^{\prime}(t), \\
& \eta_{6}=j(t)-x\left[n^{*} f^{\prime}(t)+f^{\prime \prime}(t)-2 \Omega g^{\prime}(t)\right]-y\left[n^{*} g^{\prime}(t)+g^{\prime \prime}(t)+2 \Omega f^{\prime}(t)\right]
\end{aligned}
$$

for its infinitesimal symmetries.

In order to obtain the similarity variable and the similarity transformation associated with the infinitesimal symmetries $\eta_{i}, i=1, \ldots, 6$, given in (4.1), we solve the Lagrange characteristic equation

$$
\begin{aligned}
\frac{d t}{1} & =\frac{d x}{f(t)}=\frac{d y}{g(t)}=\frac{d u}{f^{\prime}(t)}=\frac{d v}{g^{\prime}(t)} \\
& =\frac{d p}{j(t)-x\left[n^{*} f^{\prime}(t)+f^{\prime \prime}(t)-2 \Omega g^{\prime}(t)\right]-y\left[n^{*} g^{\prime}(t)+g^{\prime \prime}(t)+2 \Omega f^{\prime}(t)\right]} .
\end{aligned}
$$

Solving the characteristic equation (4.2) we obtain the similarity variables

$$
\bar{x}=x-F(t), \quad \bar{y}=y-G(t),
$$

where $F(t)=\int f(t) d t$ and $G(t)=\int g(t) d t$. Also the corresponding similarity transformations become

$$
\begin{aligned}
u= & \bar{u}(\bar{x}, \bar{y})+f(t), \quad v=\bar{v}(\bar{x}, \bar{y})+g(t), \\
p= & \bar{p}(\bar{x}, \bar{y})-x f^{\prime}(t)-y g^{\prime}(t)-\left(n^{*} x+2 \Omega y\right) f(t) \\
& -\left(n^{*} y-2 \Omega x\right) g(t)+k(t),
\end{aligned}
$$

where

$$
k(t)=\frac{1}{2}[f(t)]^{2}+\frac{1}{2}[g(t)]^{2}+\int\left[n^{*}[f(t)]^{2}+n^{*}[g(t)]^{2}+j(t)\right\} d t .
$$

Substituting the similarity transformations, (4.4), along with similarity variables (4.3) in the equations of motion (2.4)-(2.6), we find that (2.4)-(2.6) reduce to a steady state:

$$
\begin{aligned}
\bar{u} \frac{\partial \bar{u}}{\partial \bar{x}}+\bar{v} \frac{\partial \bar{u}}{\partial \bar{y}}-2 \Omega \bar{v}+n^{*} \bar{u} & =-\frac{\partial \bar{p}}{\partial \bar{x}}+\mu\left(\frac{\partial^{2} \bar{u}}{\partial \bar{x}^{2}}+\frac{\partial^{2} \bar{u}}{\partial \bar{y}^{2}}\right), \\
\bar{u} \frac{\partial \bar{v}}{\partial \bar{x}}+\bar{v} \frac{\partial \bar{v}}{\partial \bar{y}}+2 \Omega \bar{u}+n^{*} \bar{v} & =-\frac{\partial \bar{p}}{\partial \bar{y}}+\mu\left(\frac{\partial^{2} \bar{v}}{\partial \bar{x}^{2}}+\frac{\partial^{2} \bar{v}}{\partial \bar{y}^{2}}\right), \\
\frac{\partial \bar{u}}{\partial \bar{x}}+\frac{\partial \bar{v}}{\partial \bar{y}} & =0 .
\end{aligned}
$$


Therefore any steady-state solution to the equations of motion (4.5)-(4.7) can be transformed by means of (4.3) and (4.4) into a time-dependent solution involving three arbitrary functions of the time variable. Since (4.5)-(4.7) involve two independent variables, we need to apply another subgroup to reduced them into an ordinary differential equation system. For this we apply a rotation subgroup generated by the parameter $\gamma$.

4.1. Reduction via rotation group To obtain a reduction by rotation group generated by the parameter $\gamma$ in (3.2), it is best to rewrite (4.5)-(4.7) in terms of the stream function $\psi$. This function is defined so that the continuity equation, (4.7), is automatically satisfied, that is, by the relations

$$
\frac{\partial \psi}{\partial \bar{x}}=\bar{v} \quad \text { and } \quad \frac{\partial \psi}{\partial \bar{y}}=-\bar{u} .
$$

The following form of equations (4.5)-(4.7) is obtained by differentiating (4.5) with respect to $\bar{y}$ and (4.6) with respect to $\bar{x}$ and then subtracting the resulting equations:

$$
\begin{aligned}
\frac{\partial \psi}{\partial \bar{x}} & \frac{\partial^{3} \psi}{\partial \bar{y}^{3}}+\frac{\partial \psi}{\partial \bar{x}} \frac{\partial^{3} \psi}{\partial \bar{x}^{2} \partial \bar{y}}-\frac{\partial \psi}{\partial \bar{y}} \frac{\partial^{3} \psi}{\partial \bar{x} \partial \bar{y}^{2}}-\frac{\partial \psi}{\partial \bar{y}} \frac{\partial^{3} \psi}{\partial \bar{x}^{3}} \\
& +n^{*}\left(\frac{\partial^{2} \psi}{\partial \bar{x}^{2}}+\frac{\partial^{2} \psi}{\partial \bar{y}^{2}}\right)-\mu\left(\frac{\partial^{4} \psi}{\partial \bar{x}^{4}}+\frac{\partial^{4} \psi}{\partial \bar{y}^{4}}+2 \frac{\partial^{4} \psi}{\partial \bar{x}^{2} \partial \bar{y}^{2}}\right)=0
\end{aligned}
$$

In this setting, the infinitesimal transformations associated with the rotation group are

$$
\bar{x}^{*}=\bar{x}+\varepsilon \bar{y}+O(\varepsilon)^{2}, \quad \bar{y}^{*}=\bar{y}-\varepsilon \bar{x}+O(\varepsilon)^{2}, \quad \psi^{*}=\psi+O(\varepsilon)^{2} .
$$

The characteristic equations obtained from (4.10),

$$
\frac{d \bar{x}}{\bar{y}}=\frac{d \bar{y}}{-\bar{x}}=\frac{d \psi}{0},
$$

give rise to the new similarity variable $\xi=\bar{x}^{2}+\bar{y}^{2}$ and to $\psi=f_{1}(\xi)$, where $f_{1}$ is an arbitrary function of $\xi$.

In terms of $f_{1}$ and $\xi$, (4.9) becomes the ordinary differential equation

$$
n^{*} \frac{d f_{1}}{d \xi}+n^{*} \xi \frac{d^{2} f_{1}}{d \xi^{2}}-8 \mu \frac{d^{2} f_{1}}{d \xi^{2}}-16 \mu \xi \frac{d^{3} f_{1}}{d \xi^{3}}-4 \mu \xi^{2} \frac{d^{4} f_{1}}{d \xi^{4}}=0 .
$$

By rearranging and substituting $h=d\left(\xi\left(d f_{1} / d \xi\right)\right) / d \xi$ in (4.11), we get

$$
4 \mu \xi \frac{d^{2} h}{d \xi^{2}}+4 \mu \frac{d h}{d \xi}-n^{*} h=0
$$


Setting $\eta=\sqrt{\left(n^{*} / \mu\right) \xi},(4.12)$ reduces to a Bessel equation

$$
\eta^{2} \frac{d^{2} h}{d \eta^{2}}+\eta \frac{d h}{d \eta}-\eta^{2} h=0
$$

The exact solution of (4.12) is

$$
h(\xi)=a_{1} I_{0}\left(\sqrt{\frac{n^{*}}{\mu} \xi}\right)+a_{2} K_{0}\left(\sqrt{\frac{n^{*}}{\mu} \xi}\right) .
$$

To get a bounded solution at $\xi=0$, we take $a_{2}=0$. Therefore the solution is

$$
h(\xi)=\frac{d}{d \xi}\left(\xi \frac{d f_{1}}{d \xi}\right)=a_{1} I_{0}\left(\sqrt{\frac{n^{*}}{\mu} \xi}\right)=a_{1} \sum_{k=0}^{+\infty} \frac{1}{(k !)^{2}}\left(\frac{n^{*}}{4 \mu}\right)^{k} \xi^{k} .
$$

Here and hereafter $I_{v}(x)$ denotes a modified Bessel function given by

$$
I_{v}(x)=\sum_{k=0}^{+\infty} \frac{1}{k ! \Gamma(k+v+1)}\left(\frac{x}{2}\right)^{2 k+v} .
$$

Integration of (4.14) with respect to $\xi$ yields

$$
\begin{aligned}
\psi=f_{1}(\xi) & =\frac{4 a_{1} \mu}{n^{*}} I_{0}\left(\sqrt{\frac{n^{*}}{\mu} \xi}\right)+c_{1} \ln (\xi)+c_{2} \\
& =a_{1} \sum_{k=1}^{+\infty} \frac{1}{(k !)^{2}}\left(\frac{n^{*}}{4 \mu}\right)^{k-1} \xi^{k}+c_{1} \ln (\xi)+c_{2}+\frac{4 a_{1} \mu}{n^{*}}
\end{aligned}
$$

or

$$
\psi(\bar{x}, \bar{y})=a_{1} \sum_{k=1}^{+\infty} \frac{1}{(k !)^{2}}\left(\frac{n^{*}}{4 \mu}\right)^{k-1}\left(\bar{x}^{2}+\bar{y}^{2}\right)^{k}+c_{1} \ln \left(\bar{x}^{2}+\bar{y}^{2}\right)+c_{2}+\frac{4 a_{1} \mu}{n^{*}} .
$$

Solution (4.15) in terms of $\bar{u}, \bar{v}$ and $\bar{p}$ is

$$
\begin{aligned}
\bar{u}= & -\bar{y}\left\{\frac{4 a_{1}}{\sqrt{\left(n^{*} / \mu\right) \xi}} I_{1}\left(\sqrt{\frac{n^{*}}{\mu} \xi}\right)+2 c_{1} \xi^{-1}\right\} \\
= & -\bar{y}\left\{a_{1} \sum_{k=0}^{+\infty} \frac{1}{k !(k+1) !} \frac{\left(n^{*} / \mu\right)^{k}}{2^{2 k-1}} \xi^{k}+2 c_{1} \xi^{-1}\right\}, \\
\bar{v}= & \bar{x}\left\{a_{1} \sum_{k=0}^{+\infty} \frac{1}{k !(k+1) !} \frac{\left(n^{*} / \mu\right)^{k}}{2^{2 k-1}} \xi^{k}+2 c_{1} \xi^{-1}\right\} \text { and } \\
\bar{p}= & c_{3}-2 c_{1} \xi^{-1}+2 n^{*} c_{1} \arctan \left(\frac{\bar{x}}{\bar{y}}\right) \\
& +\left[(2+\Omega) c_{1} \ln ^{2}(\xi)+\left(2 a_{1}+2 \Omega+\frac{n^{*}}{2 \mu} c_{1}\right) \bar{x}^{2}+\cdots\right] a_{1},
\end{aligned}
$$


where $c_{1}, c_{2}$ and $c_{3}$ are constants to be determined from the boundary conditions and $\xi=\bar{x}^{2}+\bar{y}^{2}$. Retracing our steps back to the original variables leads us to the time-dependent solution

$$
\begin{aligned}
u= & f(t)-(y-G(t))\left\{\frac{4 a_{1}}{\sqrt{\left(n^{*} / \mu\right) \xi}} I_{1}\left(\sqrt{\frac{n^{*}}{\mu} \xi}\right)+2 c_{1} \xi^{-1}\right\}, \\
v= & g(t)+(x-F(t))\left\{\frac{4 a_{1}}{\sqrt{\left(n^{*} / \mu\right) \xi}} I_{1}\left(\sqrt{\frac{n^{*}}{\mu} \xi}\right)+2 c_{1} \xi^{-1}\right\} \text { and } \\
p= & -x f^{\prime}(t)-y g^{\prime}(t)-\left(n^{*} x+2 \Omega y\right) f(t)-\left(n^{*} y-2 \Omega x\right) g(t) \\
& +k(t)+c_{3}-2 c_{1} \xi^{-1}+2 n^{*} c_{1} \arctan \left(\frac{x-F(t)}{y-G(t)}\right) \\
& +\left[(2+\Omega) c_{1} \ln ^{2}(\xi)+\left(2 a_{1}+2 \Omega+\frac{n^{*}}{2 \mu} c_{1}\right)(x-F(t))^{2}+\cdots\right] a_{1},
\end{aligned}
$$

where $\xi=[x-F(t)]^{2}+[y-G(t)]^{2}$.

The velocity vector is $V=V_{0}+\bar{V}$, where

$$
V_{0}=(f(t), g(t)) \text { and } \bar{V}=(\bar{u}(\bar{x}, \bar{y}), \bar{v}(\bar{x}, \bar{y}))
$$

with $\bar{x}=x-F(t)$ and $\bar{y}=y-G(t)$. We observe that for any fixed time $t>0, \bar{V}$ is a rotating vector field around the centre $(F(t), G(t))$ and $V_{0}$ is the velocity of the moving centre along a line $x=F(t)$ and $y=G(t)$. Further the stream function in term of $x$ and $y$ has the form $\hat{\psi}(x, y)=g(t) x-f(t) y+\psi(\xi)$, where $\psi(\xi)$ is given in (4.15). If we choose the integral constant $c_{1}=0$ and take $\psi(\xi) \approx \xi+O(\xi)^{2}$, with $\xi \ll 1$, then the streamlines are just like circles with a modified centre and can be observed from Figure 1 (a). For the case when the integral constant $c_{1} \neq 0$, there will be a pool on the centre $(F(t), G(t))$. This can be seen from Figure 1 (b). For Figures 1 (a) and (b), the magnetic field is assumed to be weak with $\sigma=\rho=\mu=0.5$, $a_{1}=1$ and $F(t)=G(t)=t$.

\section{Conclusion}

We consider the unsteady motion of a conducting fluid in a rotating Cartesian coordinate system. Lie group theory is applied to obtain the symmetry groups and generators of the equations of motion. Then by using the subgroup generated by $f(t)$, $g(t)$ and $j(t)$ we transform the original system of partial differential equations into an ordinary differential equation. An exact solution involving a special function is obtained. The physical interpretation along with a plot of the solution is also presented. From the solution (4.17), it may be observed that the effect of electromagnetic 


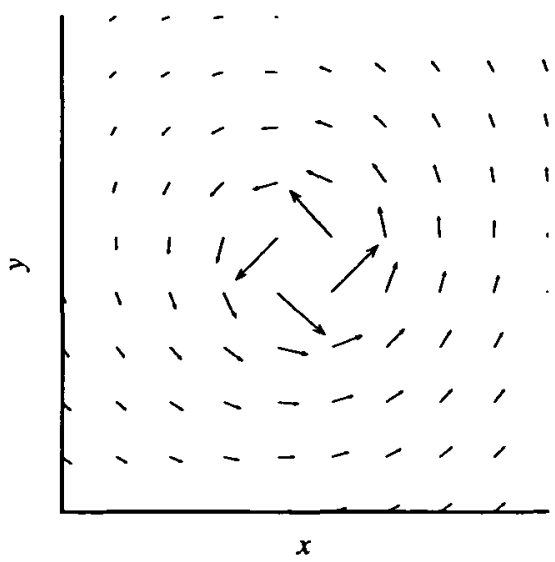

(a) $c_{1}=0$

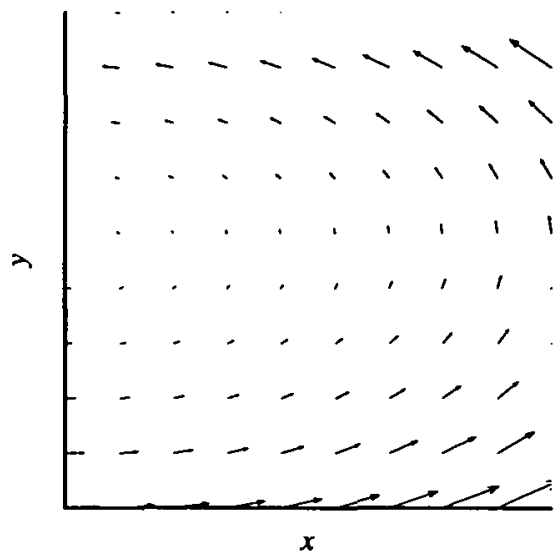

(b) $x>0\left(c_{1} \neq 0\right)$

FIGURE 1. Plots of velocity field.

force is reflected in the unsteady velocity field through $n^{*}$. Further, the result for a nonconducting fluid can be obtained by taking $B_{0}$ to be zero. Finally, it is worth mentioning that in this article we do not use the so-called small parameter assumption at all, which is however absolutely necessary for perturbation techniques. Thus the Lie group theory is shown to be very useful for the theory analysis of rotating fluid.

\section{Acknowledgements}

The authors wish to thank David Lewis Robson at the University of Science and Technology of China for his generous time in reviewing the manuscript. This project was supported by the NNSF of China (under Grants No. 10371116 and No. 10301030).

\section{References}

[1] G. W. Bluman and S. Kumei, Symmetries and differential equations (Springer, New York, 1989).

[2] L. Debnath, "On unsteady mabnetohydrodynamic boundry layers in a rotating flow", ZAMM 52 (1972) 623-634.

[3] L. Debnath, "On Ekman and Hartmann boundry layers in a rotating fluid", Acta Mech. 18 (1973) 333-340.

[4] L. Debnath, "Resonant oscillations of a porous plate in an electrically conducting rotating viscous fluid", Phys. Fluids 17 (1974) 1704-1706.

[5] L. Debnath and Mukherjee, "Unsteady multiple boundary layers on a porous plate in a rotating system", Phys. Fluids 16 (1973) 1418-1421. 
[6] K. Fakhar, R. Shagufta and Z. C. Chen, "Lie group analysis of the Navier-Stokes equations in the polar co-ordinates", IJDEA 7 (2003) 311-323.

[7] K. Fakhar, R. Shagufta and Z. C. Chen, "Lie group analysis of the axisymmetric flow", Chaos, Solitons, Fractals 19 (2004) 1261-1267.

[8] H. P. Greenspaning and L. N. Howard, "On a time dependent motion of a rotating fluid", J. Fluid. Mech. 17 (1963) 385-396.

[9] T. Hayat, A. H. Kara and E. Momoniat, "Exact flow of a third-grade fluid on a porous wall", Int. J. Non-Linear Mech. 38 (2003) 1533-1537.

[10] J. R. Holton, "The influence of viscous boundry layers on transient motions in a stratified rotating fluid", Int. J. Atoms. Sci. 22 (1965) 402-412.

[11] N. H. Ibragimov, Elementary Lie group analysis and ordinary differential equations (John Wiley \& Sons, New York, 1999).

[12] E. Momoniat, D. P. Mason and F. M. Mahomed, "Non-linear diffusion of an axisymmetric thin liquid drop: group-invariant solution and conservation law", Int. J. Non-Linear Mech. 36 (2001) 879-885.

[13] P. J. Olver, Application of Lie Groups to Differential Equations (Springer, Berlin, 1986).

[14] J. A. Shercliff, A Textbook of Magnetohydrodynamics (Pergamon, London, 1965).

[15] W. L. Siegmann, "The spin-down of a rotating stratified fluids", J. Fluid Mech. 47 (1971) 689-701.

[16] G. Walin, "Some aspects of a time dependent motion of a stratified rotating fluid", J. Fluid Mech. 36 (1969) 289-300.

[17] M. Yürüsory, M. Pakdemirli and Ö. F. Noyan, "Lie group analysis of a creeping flow of a second grade fluid", Int. J. Non-Linear Mech. 36 (2001) 955-960. 